\title{
GPPS-TC-2019-XXXX
}

\section{BIFURCATION DODGE: AVOIDANCE OF A THERMOACOUSTIC INSTABILITY UNDER TRANSIENT OPERATION}

\author{
Giacomo Bonciolini; Nicolas Noiray \\ CAPS Laboratory - Mechanical and Process Engineering Department \\ ETH Zürich, 8092, Zurich, Switzerland
}

\begin{abstract}
Dynamical systems can exhibit critical transitions if one of their parameter is varied. These critical transitions often lead to undesirable or dangerous states, which can cause the failure of the system. In some cases, there is only a limited range of the parameter value leading to that dangerous condition, while the system runs safely otherwise. In this study, we present experimental results regarding a thermoacoustic system subject to two consecutive and mirrored supercritical Hopf bifurcations: the system exhibits high amplitude oscillations for the central part of the considered parameter range. Changing rapidly the bifurcation parameter, we were able to dodge this dangerous condition, avoiding any stress for the system. A low-order model of the system was then developed, to assess what is the risk associated with this "crossing of the danger zone" as a function of the rapidity at which that zone is crossed.
\end{abstract}

\section{INTRODUCTION}

Natural and social scientists often face complex dynamical systems that markedly change their behaviour according to the operating conditions. This observation is translated mathematically into the concept of bifurcation; a dynamical system undergoes a bifurcation when, for a small change of one of its parameters, the output of the system is modified in its quality and quantity [1]. Some examples of this concept can be found in recent publications. In [2], a change in atmospheric $\mathrm{CO}_{2}$ concentration is expected to cause coral reef extinction; [3] investigates the emergence of consumption demands limit cycles and the effects on economy as a function of environment pollution; in [4],

\footnotetext{
giacomob@ethz.ch

†noirayn@ethz.ch
}

This work is licensed under a Creative Commons Attribution 4.0 International License Creative Commons Attribution-NonCommercial-NoDerivatives 4.0 International License the influence of different parameters on climate and ecosystems catastrophes is reviewed; in [5], a value of the Reynolds number over a certain threshold causes a supercritical Hopf bifurcation of the flow velocity in a helical pipe. In all of these examples, and in many other more, the system bifurcates to undesirable and/or dangerous states, which can be extremely detrimental for the integrity of the system. That is the reason why the development of tools that detect precursors of a bifurcation is an active research area [6-15].

In some cases, the system is in danger for a certain interval of the bifurcation parameter's value, while it runs safely otherwise (see for instance [16]). This is the situation we will treat in the present study, with the additional complication of considering a system with a stochastic output. This feature is common to many systems, for which the output is a random quantity characterized by a certain probability density distribution. The considered situation is depicted in fig. 1a, in a stochastic bifurcation diagram fashion. The abscissa expresses the bifurcation parameter, which can assume any value between two extrema. This parameter determines the output of the system, which is presented in the ordinate in terms of its amplitude. As discussed, we will deal with a stochastic system; therefore that output amplitude is described in terms of its probability density function (PDF): the contour map represents the likelihood of any state, with darker hues corresponding to more probable ones. The red line represents the mean of the distribution at each operating condition. This example system has a non monotonic behaviour; it gives a small amplitude output for low and high values of the control parameter, while it provides high amplitude outputs for intermediate values. One can imagine that the system can be imperilled if its output exceeds a certain safety level, marked with a black dashed line in the figure. Therefore, considering where the mean amplitude 

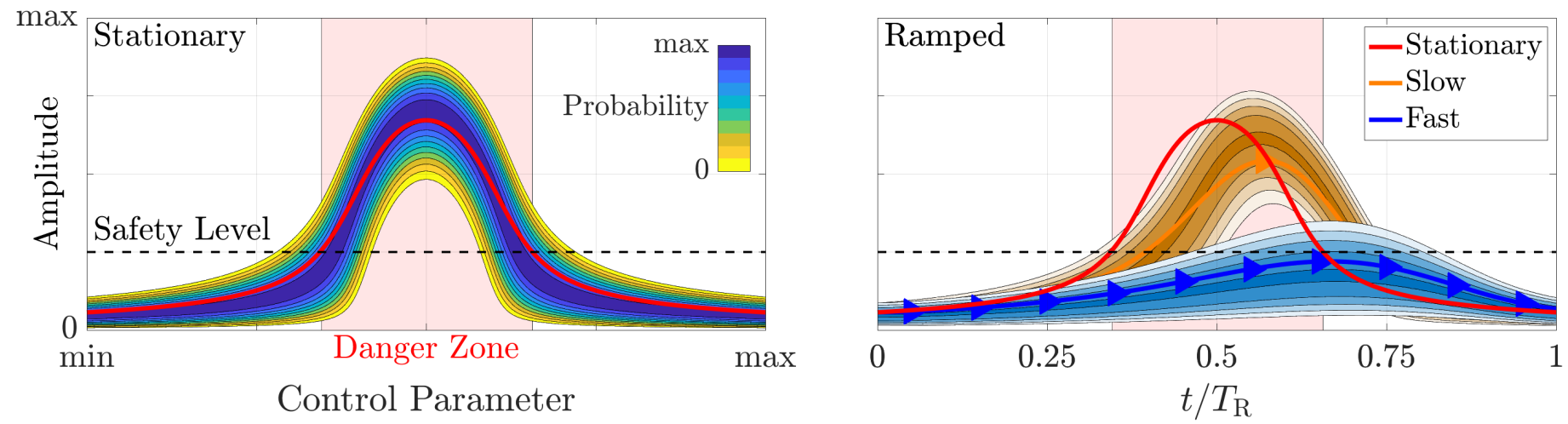

FIGURE 1. Context of the paper. Left: amplitude bifurcation diagram of two consecutive and mirrored stochastic supercritical bifurcations. Given a safety level for the amplitude, a "Danger Zone" for the bifurcation parameter is determined. Right: possibility of dodging the high-amplitude zone of the bifurcation, by ramping fast the bifurcation parameter.

crosses this level, a "Danger Zone" for the control parameter is identified in the middle of the examined range.

One might question whether is possible to pass across the danger zone without incurring in high amplitude outputs. In this paper, we show that this is indeed possible, provided that the control parameter is ramped fast enough through the danger zone. We will define as "Bifurcation Dodge" this avoidance of the high amplitude output via a fast ramping of the control parameter . This concept is illustrated in fig. 1b. This plot represents the evolution in time of the system's output amplitude PDF, under two ramps of the control parameter having different duration $T_{\mathrm{R}}$. While a slow ramp is ineffective in avoiding the transition to high-amplitude states (orange contour), a fast ramp (blue contour), is expected to enable a bifurcation dodge, as the state stays on average below the safety level. This can happen because the system experiences in the case of a fast ramp a certain "inertia"; the time needed to reach the target state of the current (ramping) condition is longer than the ramp duration, and therefore the system's output jumps from one low-amplitude attractor to the other, without being attracted to the high-amplitudes of the danger zone. In the following section, we introduce a system that can feature a non-monotonic dependence of the output amplitude on the control parameter, and that we used to demonstrate the feasibility of a bifurcation dodge. In section 3.2, we will describe a non-linear oscillator model of that system. In section 4.1, the results of the experiment showing the bifurcation dodge will be presented. In section 4.2, it will be shown how the model is able to reproduce the observed dynamics. Finally, in section 5 the model will be used to assess the probability of dodging the bifurcation as a function of the system's parameters.

\section{THERMOACOUSTIC INSTABILITIES}

Thermoacoustic instabilities are a challenging problem encountered in many practical combustion systems, such as heavy- duty gas turbines, aeronautical or rocket engines [17]. This phenomenon arises due to the coupling between unsteady heat release rate and the acoustic field that is present in the combustor volume [18]. Under certain conditions, this interaction becomes constructive, leading to extremely high acoustic pressure oscillations. This acoustic field loads the mechanical components and can lead to fatigue failures. Therefore, the thermoacoustic stability of a new combustor must be assessed in the design phase, to avoid any unstable operation. The stability depends on the geometry of the system, but also on the operating conditions. Regarding the latter, the stability of the system often does not vary monotonically with the value of the operating parameters. Often, the non-monotonic stability behaviour is associated with a change of the phase lag between the flame response to acoustically-triggered flow perturbations and the local acoustic field [19].

Such a non-monotonic behaviour was already observed in our previous study [20]. In that case, however, the driver of the instability was none of the aforementioned mechanisms; the system was becoming unstable due to a morphology change of the flame. This phenomenon was caused by a strong interaction between the flame and the walls of the combustor; it was found that the real bifurcation parameter was the temperature of the walls. As a consequence, it was not possible to dodge the bifurcation when ramping: as explained extensively in that paper, due to this peculiar instability mechanism, one cannot directly control the ramp speed and pass rapidly through the unstable region, due to the walls' thermal inertia.

In the present study, to avoid this mechanism, we modified the configuration of the system, as depicted in fig. 2. The panel (a) represents the configuration of the old study [20]. The current configuration (b) has the following main differences:

- The combustion chamber diameter was increased from 70 to $100 \mathrm{~mm}$. In this way, the flame is less confined and it is 

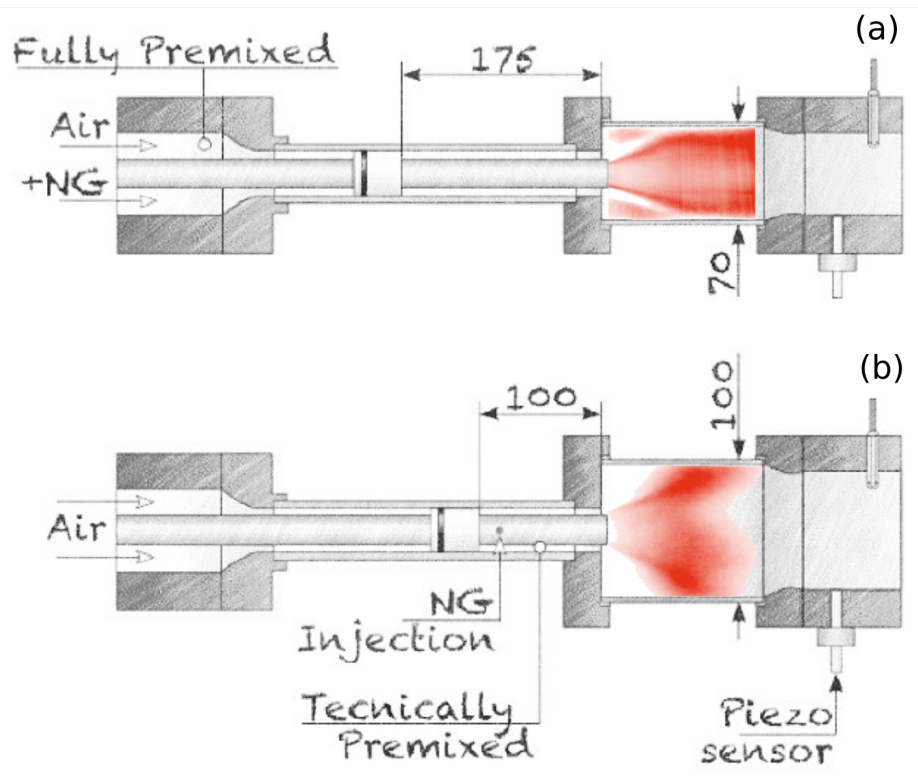

FIGURE 2. Comparison between (a) the geometry of the combustor configuration used in [20], and (b) the one used in the present study.

expected to interact less with the walls.

- The swirler was moved $75 \mathrm{~mm}$ closer to the lance termination

- The combustor is not operated in fully premixed mode, but in technically premixed mode, with natural gas injection downstream of the swirler.

Other general details about this combustor architecture and how it is operated are provided in [20]. In the following section we present the results of the mapping of this new configuration, and we show how this exhibits a non-monotonic thermoacoustic bifurcation as a function of one control parameter.

\section{STATIONARY OPERATION}

The combustor presented in fig. 2b can run at different operating conditions, changing the amounts of air and natural gas (NG) that take part to the combustion. As discussed, the system might experience thermoacoustic instabilities for some of these conditions. The acoustic pressure was recorded by a watercooled Kistler piezoelectric sensor type 211B2 placed after the flame in the combustion chamber. Fig. 3 represents a map of the acoustic pressure root mean square level $\left(p_{\text {rms }}\right)$ as a function of the two operating parameters that were varied to explore the behaviour of the system: the equivalence ratio $\phi$ and the natural gas mass flow rate $\dot{m}_{\mathrm{NG}}$. The explored range is $\phi \in[0.69 ; 1.1]$ and $\dot{m}_{\mathrm{NG}} \in[0.7 ; 0.9] \mathrm{g} / \mathrm{s}$, corresponding to a thermal power ranging from 26.6 to $34.2 \mathrm{~kW}$. Analyzing this map, one can notice how the system exhibits high amplitude acoustic oscillation in

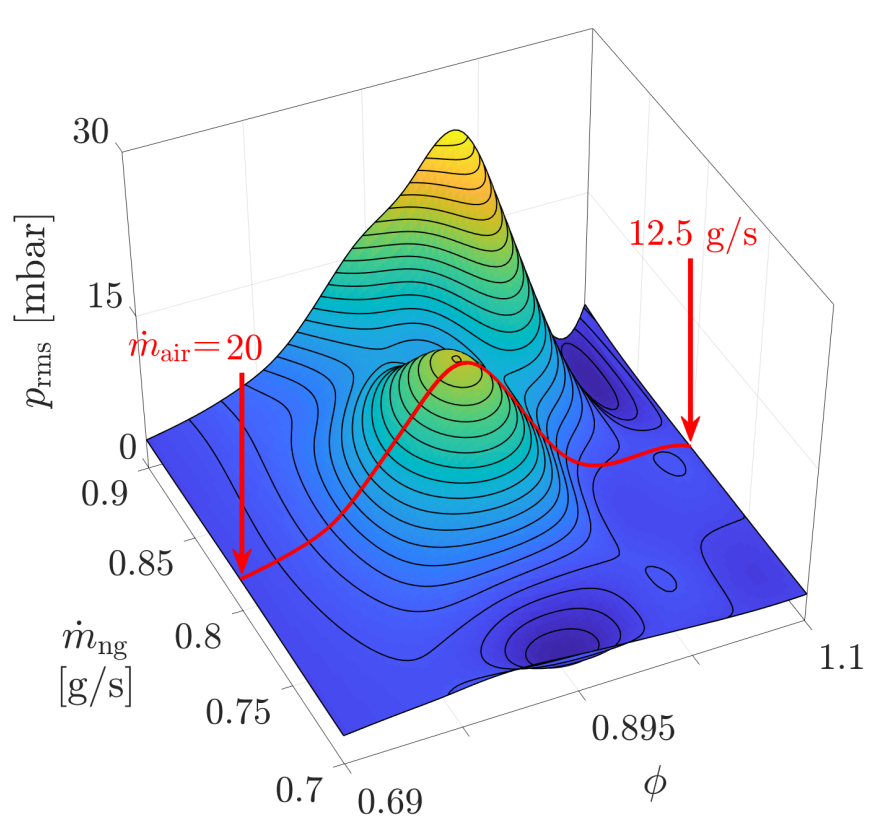

FIGURE 3. Root mean square of the acoustic pressure in the combustion chamber, as a function of the operating condition, defined by the natural gas mass flow rate $\dot{m}_{\mathrm{NG}}$ and the equivalence ratio $\phi$. The red line is the path that is analyzed in the remainder of this study.

two unstable regions, one located at intermediate $\dot{m}_{\mathrm{NG}}$ and $\phi$ and the other at high $\dot{m}_{\mathrm{NG}}$ and $\phi$.

\subsection{Thermoacoustic bifurcation}

In the remainder of this study we will focus on the bifurcation highlighted with a red line on the map of fig. 3: the combustor was operated with a constant $\dot{m}_{\mathrm{NG}}$ of $0.8 \mathrm{~g} / \mathrm{s}$, varying the air mass flow rate $\dot{m}_{\mathrm{AIR}}$-which serves as bifurcation parameterfrom 12.5 to $20 \mathrm{~g} / \mathrm{s}$. In that way, the whole equivalence ratio range was covered.

The detailed analysis of this bifurcation is shown in fig. 4. The data presented here are obtained post-processing 2 minutes long acoustic pressure $p(t)$ acquisitions at stationary condition. In the upper panel, the power spectral density (PSD) $S_{p p}$ at each operating condition is plotted. One can notice how a mode at circa $250 \mathrm{~Hz}$ is present all over the examined range. In the central part of the range, the quality factor of the $250 \mathrm{~Hz}$ peak increases, and at the same time a peak at the first harmonic appears. These facts indicate the emergence of a thermoacoustic limit cycle associated to the mode: the system oscillates at the mode's frequency with high amplitude. In the present condition, where one mode dominates the thermoacoustic dynamics, the acoustic pressure 


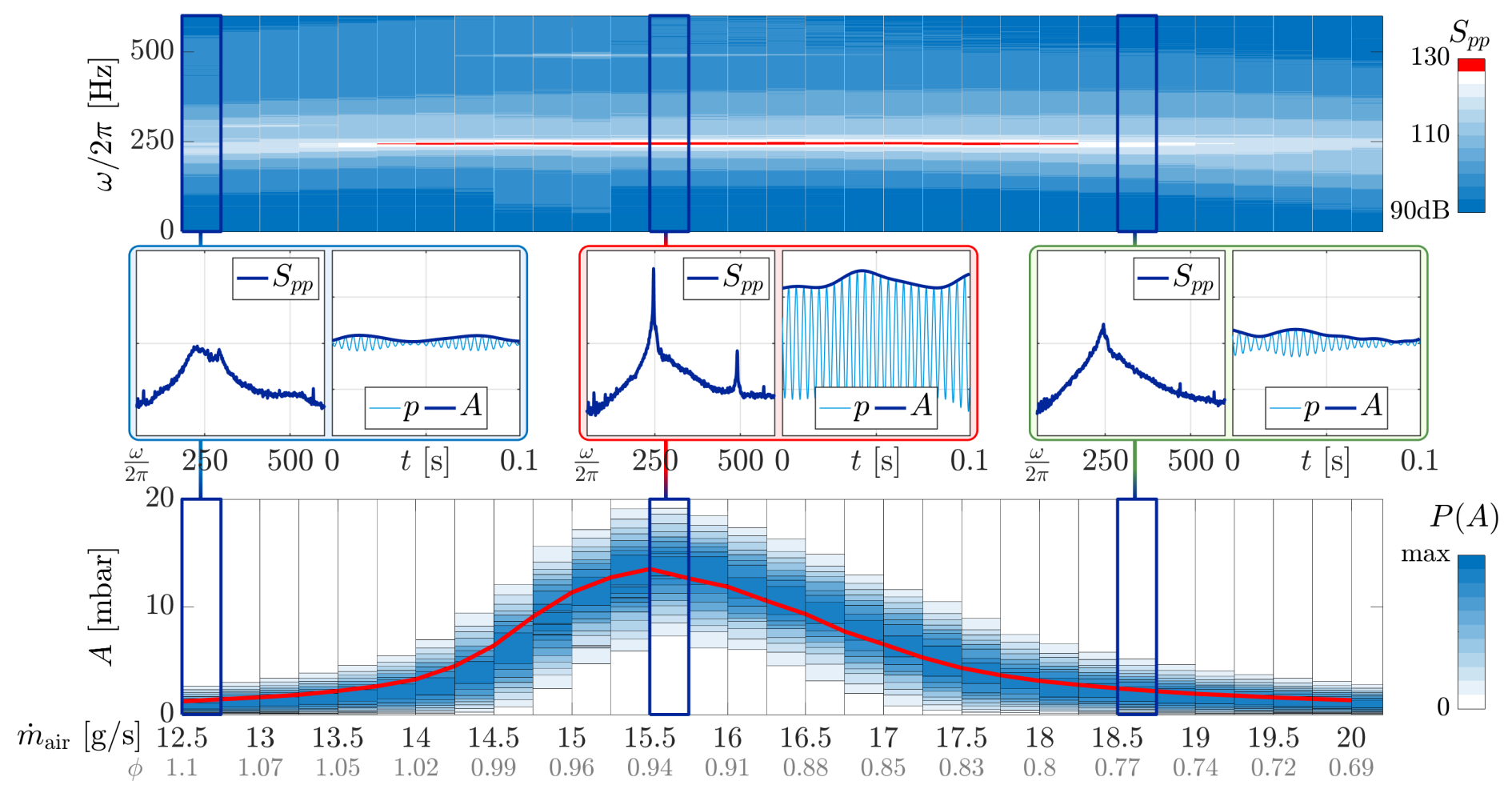

FIGURE 4. Stationary operations of the combustor at $\dot{m}_{\mathrm{NG}}=0.8 \mathrm{~g} / \mathrm{s}$, as a function of $\dot{m}_{\mathrm{AIR}}$. Top: Acoustic pressure power spectral density. Bottom: Acoustic pressure oscillation amplitude probability density function. Center: details of spectra and time traces of three selected operating conditions: $\dot{m}_{\mathrm{AIR}}=12.5,15.5,18.5 \mathrm{~g} / \mathrm{s}$.

evolution can be approximated as $p(t) \approx A(t) \cos \left(\omega_{0} t+\varphi(t)\right)$, where $\omega_{0}$ is the mode angular frequency and $\varphi(t)$ and $A(t)$ two slowly-varying quantities representing the stochastic fluctuations of oscillation phase and amplitude. These two quantities are fluctuating because this system is subject to a stochastic forcing exerted by turbulence, which relentlessly drives the system away from its deterministic attractor. The oscillation amplitude $A(t)$ is therefore a random variable with distribution $P(A)$. In the lower panel $P(A)$ is presented (contour map) together with the distribution mean (red line) as a function of $\dot{m}_{\text {AIR }}$. This representation confirms what was already deducible from the power spectral density plot. The system undergoes two consecutive and mirrored supercritical Hopf bifurcations. At low $\dot{m}_{\mathrm{AIR}}$, the system is linearly stable and the recorded fluctuations are only due to the turbulence stochastic forcing. At intermediate air mass flow rates, the system loses its linear stability: a stochastically perturbed limit cycle is established. For high $\dot{m}_{\mathrm{AIR}}$, the system is stable again: only small damped oscillations are registered. These three steps are exemplified by the three points $\dot{m}_{\text {AIR }}=12.5$ $\mathrm{g} / \mathrm{s}$ (stable), $15.5 \mathrm{~g} / \mathrm{s}$ (unstable), $18.5 \mathrm{~g} / \mathrm{s}$ (stable) in the central row of fig. 4, where their PSD and acoustic time traces are plotted.

This behaviour is analogous to the one presented in [20]. In this case, however, the instability is not accompanied by a flame topology transition, as displayed in fig. 5. These high speed images were recorded at a frame rate of $5 \mathrm{kHz}$ with a CMOS camera (Photron FASTCAM SA-X2) coupled to a high-speed intensifier (LaVision HS-IRO) equipped with a $100 \mathrm{~mm} \mathrm{f} / 2.8 \mathrm{UV}$ lens (Cerco, aperture f\#6) and a bandpass interference filter for $\mathrm{CH}^{*}$ (Edmund, transmission $>90 \%$ at $430 \mathrm{~nm}$, FWHM $10 \mathrm{~nm}$ ). In the top row, it is shown the mean flame shape at the three operating conditions $\dot{m}_{\text {AIR }}=12.5,15.5,18.5 \mathrm{~g} / \mathrm{s}$. For the unstable case $\dot{m}_{\mathrm{AIR}}=15.5 \mathrm{~g} / \mathrm{s}$, more details are provided in the bottom panel. Each column represents one phase $\theta$ of the acoustic cycle $p(t)=A(t) \cos (\theta(t))$, and it is split in two rows; the top row shows the instantaneous snapshots of the flame $\mathrm{CH}^{*}$ chemiluminescence, the bottom one is the corresponding phase-avareged flame shape. One can observe how the flame, as a consequence of the variation of the mean flow velocity and of $\phi$ (and hence of turbulent flame speed), changes angle across the shown operating conditions. However, the flame stays in any case with a V-shape and does not experience a transition to M-shape as in [20].

This fact increases the chances that $\dot{m}_{\mathrm{AIR}}$ is the actual bifurcation parameter and that it will be possible to dodge the bifurcation if that parameter is ramped fast enough. 


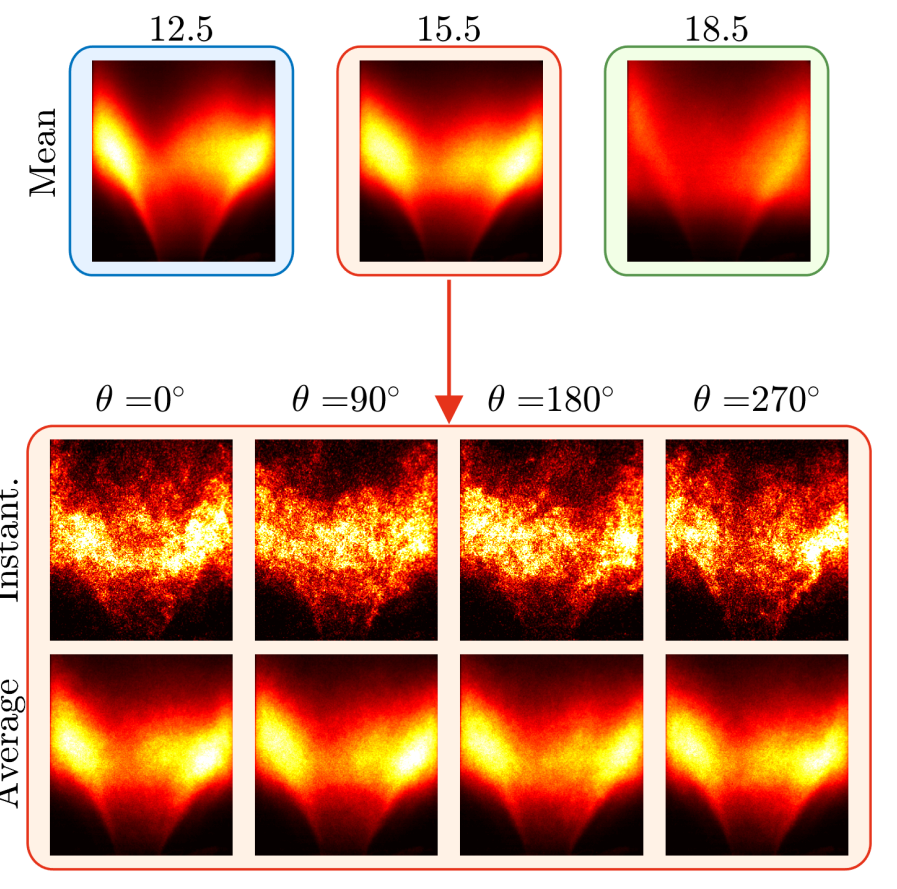

FIGURE 5. Top: High-speed camera acquisitions of the flame mean $\mathrm{CH}^{*}$ chemiluminescence at three different operating conditions. From left to right: $\dot{m}_{\mathrm{AIR}}=12.5 ; 15.5 ; 18.5 \mathrm{~g} / \mathrm{s}$. Bottom: Detail of the $\dot{m}_{\mathrm{AIR}}=$ $15.5 \mathrm{~g} / \mathrm{s}$ case, with an instantaneous snapshot and the corresponding phase-averaged $\mathrm{CH}^{*}$ chemiluminescence at four different phases $\theta$ of the acoustic cycle $p(t)=A(t) \cos (\theta(t))$.

\subsection{Model and Identification of parameters}

Before moving to the transitory dynamics, we introduce a low-order model of the system that will help in understanding the observed physics. In the current setup, as already pointed out, the combustor experiences two consecutive and mirrored supercritical bifurcations, when increasing the air mass flow rate. An established model for this scenario is the stochastically forced Van der Pol oscillator (VDP) [21-23]. Its dynamical equation reads:

$$
\ddot{p}+\omega_{0}^{2} p=\left[2 v-\kappa p^{2}\right] \dot{p}+\xi(t),
$$

where $p$ is the state of the oscillator, mimicking the acoustic pressure in the combustor, $\omega_{0}$ the natural angular frequency (in our case the considered mode's one), $v$ the linear growth rate, which determines with its sign the linear stability of the system, $\kappa$ a positive constant that allows for the saturation of the growth and the establishment of a limit cycle, $\xi(t)$ a gaussian white noise of intensity $\Gamma$ that mimicks the stochastic forcing exerted by turbulence [24]. The solutions of this equation, given the parameters, will assume the form of a sinusoid with slowly-varying ampli-
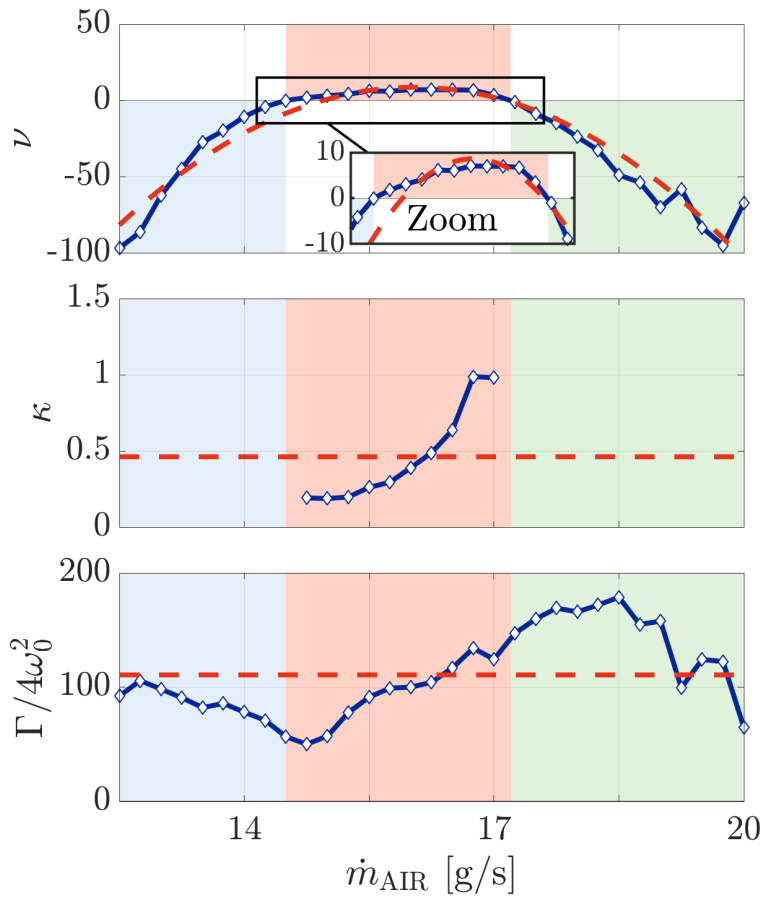

FIGURE 6. Identification of the low-order model parameters. The linear growth rate $v$, the cubic saturation constant $\kappa$ and the noise intensity $\Gamma$ are identified with the output-only method proposed in [26]. The background colors of these three panels indicate the linear stability of the system, as deduced from the sign of $v$. The dashed lines indicate the values adopted for $v\left(\dot{m}_{\mathrm{AIR}}\right), \kappa, \Gamma$, in the simulations performed in section 4.2.

tude $p(t)=A(t) \cos \left(\omega_{0} t+\varphi(t)\right)$, as for the experimental time traces. It is possible to fit the experimental data to this model and, via output-only identification tools [25], retrieve the values of the parameters in (1) at each operating condition. Figure 6 presents the result of the identification process, performed with the robust identification algorithm proposed in [26]. One can notice how the observed non-monotonic dependence of the acoustic amplitude on $\dot{m}_{\mathrm{AIR}}$ corresponds to a non-monotonic trend of the identified linear growth rate $v$. At low and high $\dot{m}_{\mathrm{AIR}}$, this parameter is negative, and therefore the system is linearly stable, while for intermediate values $v$ is positive, and the system exhibits a limit cycle. The dependence of linear growth rate on the air mass flow rate can be approximated with a cosine

$$
v\left(\dot{m}_{\mathrm{AIR}}\right)=n_{1} \cos \left(n_{2} \dot{m}_{\mathrm{AIR}}\right)+n_{3},
$$

while for $\kappa$ and $\Gamma$, although they present in reality a dependence on $\dot{m}_{\mathrm{AIR}}$, we will consider for simplicity their mean value in the range (see the red dashed lines in fig. 6). 
After this characterization of the system at stationary conditions, we move into the next section, where the dynamics under transient operation is experimented and analyzed.

\section{RAMP AND BIFURCATION DODGE}

In this section, the dynamics of the system under transient operation is presented. Thermoacoustic dynamics under transient conditions is a topic that has received only limited attention, with some few exceptions like [20,27,28]. This despite the fact that real machines are often operated with varying operating conditions, and that this variation is sometimes performed at a fast rate, for instance in aero-engines.

\subsection{Experimental results}

To perform the bifurcation parameter ramp test, an automatic controller was set to repeat 50 times a progressive opening of the air valve, such that $\dot{m}_{\mathrm{AIR}}$ increased from 12.5 to $20 \mathrm{~g} / \mathrm{s}$ in a quasi-linear fashion during each ramp. The ramps duration is $T_{\mathrm{R}}$. The corresponding recorded acoustic time traces of these 50 repetitions of the experiment were used to extract the statistic of this transient process. The results are presented in fig. 7a. In these plot one can see the evolution in time of the acoustic amplitude PDF $P(A ; t)$. For ramp times longer than $5 \mathrm{~s}$, the transient PDF resembles the stationary one: no inertial effects are triggered. In the first row of the figure, the statistic of a ramp lasting $T_{\mathrm{R}}=1.19 \mathrm{~s}$ is reported. One can notice how the "inertia" of the system starts to play a role: the development of the instability is delayed and the maximum attained amplitude lowered (see the stationary mean amplitude plotted for reference as a red line). In the second row, the result of a test with a fast ramp lasting $T_{\mathrm{R}}=0.16 \mathrm{~s}$ is plotted. In this case, the onset of instability is almost suppressed: the acoustic level stays low and the bifurcation is dodged.

\subsection{Simulations results}

We reproduced the dynamics observed in the experiment with our low-order model, presented in the previous section. The control parameter is ramped linearly over time at a certain rate $R: \dot{m}_{\mathrm{AIR}}(t)=\dot{m}_{0}+R \cdot t . R$ is chosen to cover the whole $\dot{m}_{\mathrm{AIR}}$ span in a time $T_{\mathrm{R}}$. As a result, the linear growth rate of the model, whose dependence on $\dot{m}_{\mathrm{AIR}}$ was approximated with a cosine (see (2)), varies over time: $v(t)=v\left(\dot{m}_{\mathrm{AIR}}(t)\right)$.

To obtain the statistic of the process, one can simulate many realizations of (1) with this time-varying $v$ (Monte Carlo method). A more elegant and computationally cheap method is to solve the Fokker-Planck equation (FPE) associated with the oscillation amplitude stochastic process and directly obtain the evolution in time of the $A$ PDF $P(A ; t)$ :

$$
\partial_{t} P=-\partial_{A}[\mathscr{F}(A, t) P]+\left(\Gamma / 4 \omega_{0}^{2}\right) \partial_{A A} P
$$

where $\mathscr{F}(A, t)=A\left[v(t)-(\kappa / 8) A^{2}\right]+\Gamma /\left(4 \omega_{0}^{2} A\right)$. Note how $v$ is in this case a function of time, and specifically it is set to reproduce the cosine dependence identified from the stationary experiment. All the other constants are set to their identified mean values, as indicated in fig. 6. This last simplification does not have a significant impact on the solution: if the FPE is solved with varying $\kappa$ and $\Gamma$ coefficient, the result is not qualitatively different. The initial condition for the FPE is the stationary PDF $P_{\infty}(A)$ at the initial state, which is a function of the system's parameters and of the initial growth rate, and which can be derived analytically ${ }^{1}$. The solutions of the FPE, for the same $T_{\mathrm{R}}$ of the experimental counterparts, are presented in fig. 7b. One can appreciate how these two simulation resembles the experimental result. Even in this case, a bifurcation dodge is observed if the bifurcation parameter is ramped fast.

One should notice how the examined ramp times $T_{\mathrm{R}}$ are extremely short and far from the considerably longer ones adopted in real machines when changing operating conditions. The possibility of dodging the bifurcation is determined by the competition between the ramp time $T_{\mathrm{R}}$ and the oscillation growth characteristic time, which is proportional to the inverse of the system's linear growth rate: $T_{v} \propto 1 / v$. When $T_{\mathrm{R}}<T_{v}$, the bifurcation is crossed faster than how fast the oscillations can grow and a bifurcation dodge is possible.

In the following section, with the help of the low-order model, we will examine what is the likelihood of having a bifurcation dodge, as a function of the ramp time and of the peak linear growth rate. We will then provide a criterion to estimate the damage inflicted to the system when ramping across the "danger zone".

\section{RISK ANALYSIS}

In this section we present the results of two analyses performed with the stochastic low order model. The first aim is to assess what is the probability of being able to dodge the bifurcation, according to the speed at which one ramps across it and to the maximum linear growth rate attained by the system. In second instance, we want to estimate what is the harm for the system in case the dodge is only partial and the output reaches high amplitudes.

In both of the cases, we established a safety level. This level represents an amplitude of the output below which the system is not solicited and does not experience any damage. As discussed, we are dealing with a stochastic system, therefore all the risk assessment will be made in a probabilistic sense.

\footnotetext{
${ }^{1} P_{\infty}(A ; t=0)=\mathscr{N} A \exp \left[4 \omega_{0}^{2} / \Gamma\left(v_{0} A^{2} / 2-\kappa A^{4} / 32\right)\right]$, where $v_{0}=v(t=0)$ and $\mathscr{N}$ is a normalization constant. See [24] for more details.
} 
a) Experiments
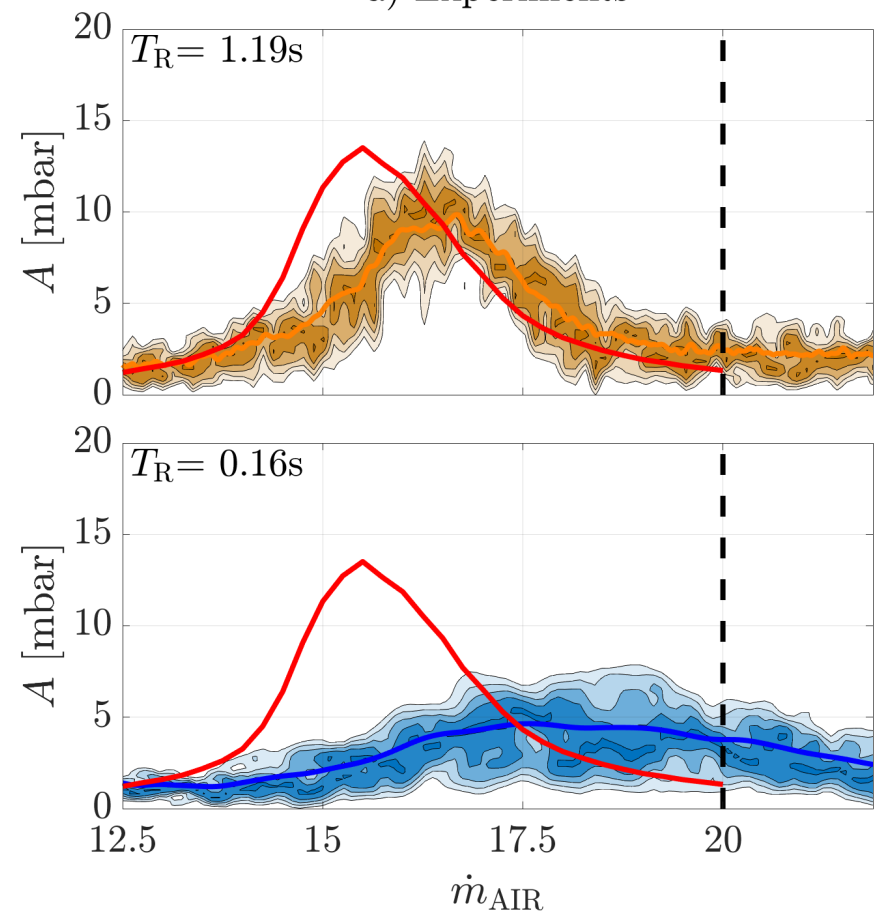

b) Simulations
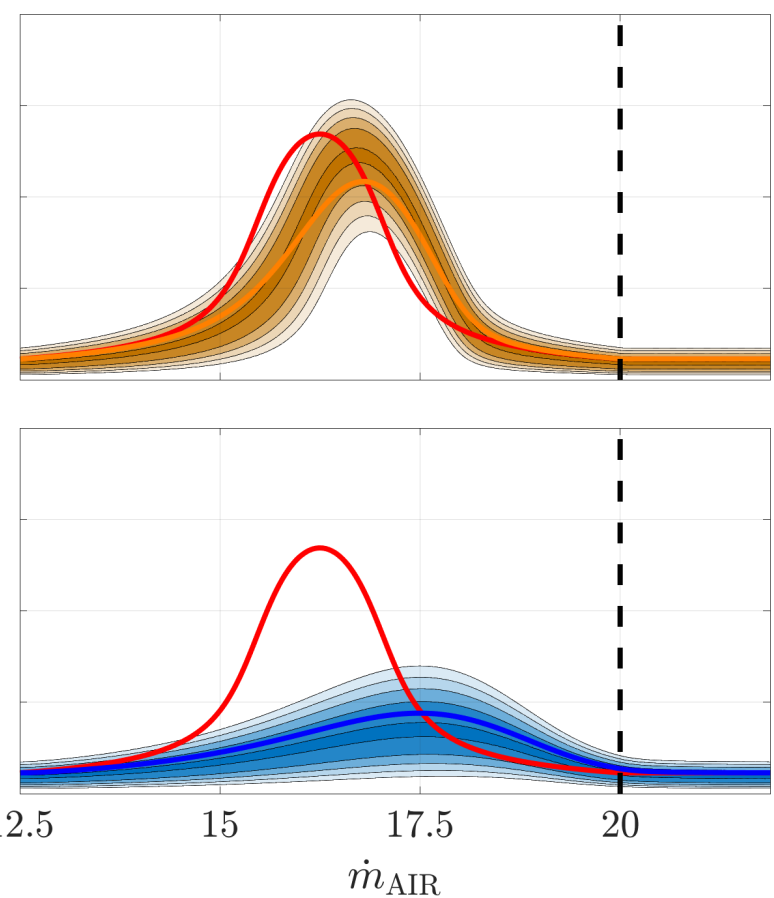

FIGURE 7. Amplitude PDF evolution under transient operation. Left: experiments performed ramping $\dot{m}_{\mathrm{AIR}}$. Right: solution of the unsteady FPE (3), with $v$ varying according to the cosine dependence on the control parameter (2), which was identified from the experiments (see fig. 6). In both cases, the ramp is performed in the two indicated ramps time $T_{\mathrm{R}}$. The red lines represent the reference stationary mean amplitude.
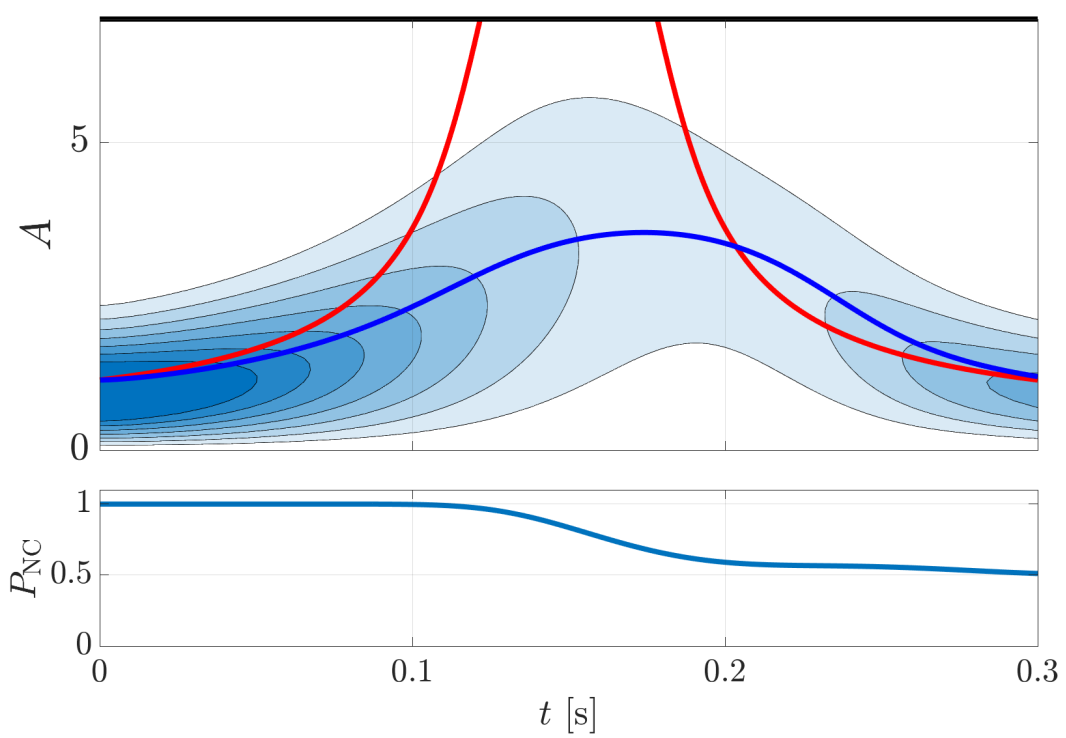

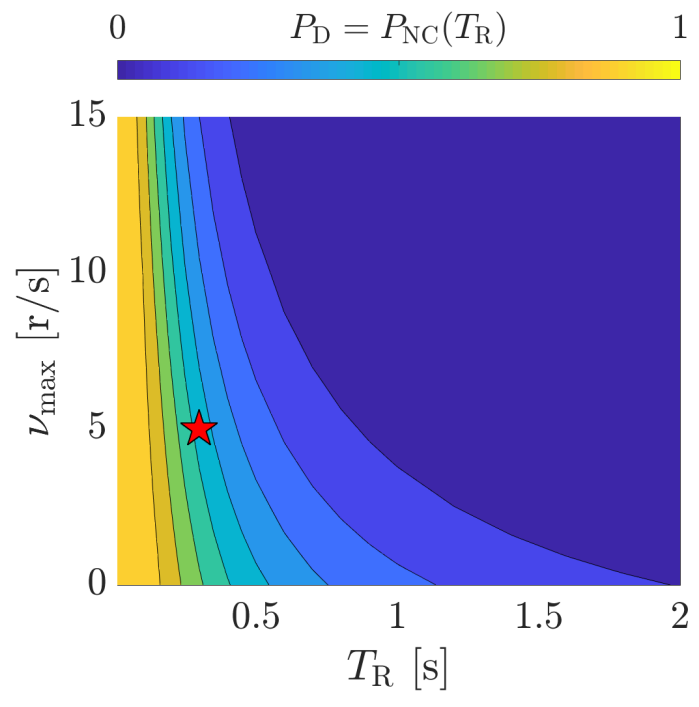

FIGURE 8. Calculation of the probability of dodging the bifurcation $P_{\mathrm{D}}$. Left: example of calculation, performed solving the FPE with an absorbing boundary condition. On top $P(A ; t)$, on the bottom $P_{\mathrm{NC}}(t)$, the probability of not having crossed the safety level before time $t$. Right: summary map of the dodge probability $P_{\mathrm{D}}=P_{\mathrm{NC}}\left(T_{\mathrm{R}}\right)$, as a function of ramp time $T_{\mathrm{R}}$ and maximum linear growth rate attained during the ramp $v_{\mathrm{max}}$. 


\subsection{Bifurcation dodge probability}

With this test we computed the probability of not crossing the safety level, which is intended as the probability of dodging the bifurcation. This is essentially an escape problem, which is often addressed in random processes studies [29-32]. This problem can be tackled in the current scenario by solving the FPE with an absorbing boundary condition at the safety level, as described in [28]. In essence, the ramping process makes the amplitude PDF drift and diffuse, and thanks to this special boundary condition, all the probability that hits the safety level is subtracted from the domain. At each instant, what is left in the domain is the probability $P_{\mathrm{NC}}(t)=\int_{0}^{\mathrm{SL}} P(A ; t) \mathrm{d} A$ of not having crossed the boundary before that time. The dodge probability can be therefore expressed as the probability of not having crossed the safety level at the end of the $\operatorname{ramp} P_{\mathrm{D}}=P_{\mathrm{NC}}\left(T_{\mathrm{R}}\right)$. The results of this test are presented in fig. 8. In the upper-left panel the result of the FPE simulation for one particular ramp rate and maximum linear growth rate $v_{\max }$ is plotted. The upper limit of the graph is the absorbing boundary. In the bottom-left panel, one can see how the probability of not having crossed decreases monotonically, at higher rates when the drift brings the probability close to the safety level. The result of this simulation is reported in the map at the right of the figure (red star). This map represents the dodge probability $P_{\mathrm{D}}$, for different combination of $T_{\mathrm{R}}$ and $v_{\max }$. The linear growth rate at the initial state and so the initial PDF is the same for all the points in the map and coincident with the one identified from the experiments. One can notice how if $v_{\max }$ is higher, and therefore the characteristic oscillation growth time shorter, a faster ramp is required to dodge even partially the bifurcation. From this map it is clear how difficult it is, even for not extremely high $v_{\max }$ values, to dodge completely the bifurcation. Therefore, in a real setup, it is expected to cross the safety level, at least with a certain probability, even if the ramp is performed at a very high rate.

In the next test we therefore provide a tool to quantify the potential damage that is caused by the crossing of the safety level.

\subsection{Damage quantification}

In this case we computed the harm inflicted to system when the amplitude of the oscillations overcomes the safety level during the ramping. This assessment is of primary importance for systems which are designed to resist to fatigue. What damages the system is the combination of the stress intensity and the number of cycles over the fatigue limit (which can be assimilated to our safety level). Therefore we computed the damage in terms of over-safety-level energy: during the ramp the system state crosses two times the safety level, the first time going from a safe operation to the dangerous high-amplitude one, the second when coming back to the safe state. Therefore an over-safetylevel time interval $\Delta t_{\mathrm{SL}}$ can be defined. The energy released dur-

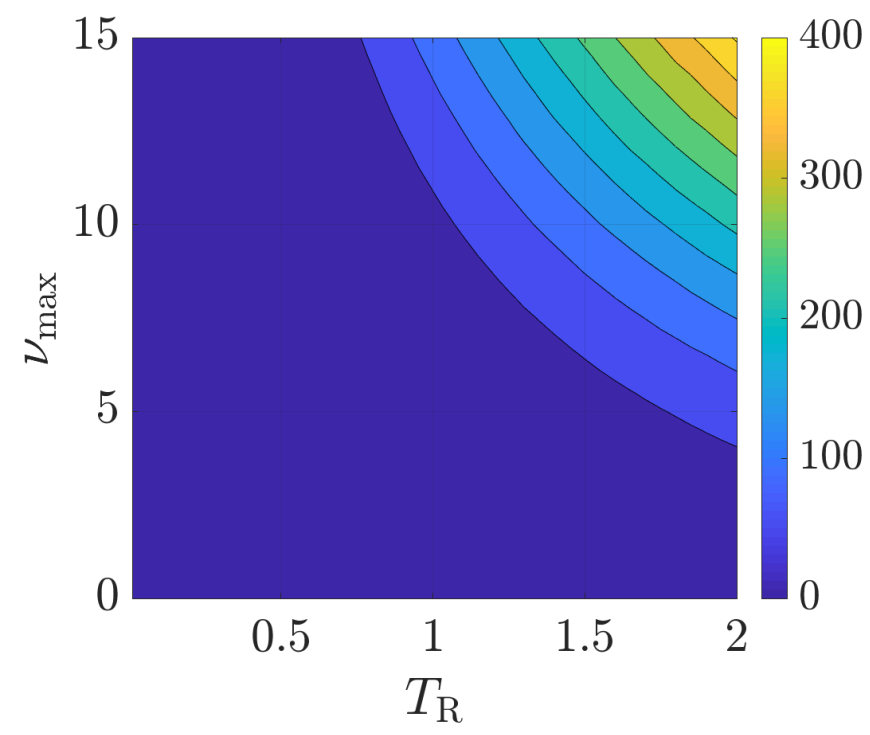

FIGURE 9. Energy over the safety level, as defined in (4), as a function of ramp time and maximum linear growth rate.

ing this interval is therefore:

$$
E=\int_{\Delta t_{\mathrm{SL}}} A(t)^{2} \mathrm{~d} t
$$

Figure 9 is a map of this quantity as a function of $T_{\mathrm{R}}$ and $v_{\max }$. The damage is more intense for higher $v_{\max }$, since, as already discussed analyzing the previous test about the dodge probability, for the same ramp time the probability of dodging is less and the attained amplitude higher. For a certain $v_{\max }$, the harm is more intense for slow ramping, since the dodge probability decreases and the time spent over the safety level is longer.

\section{CONCLUSIONS}

In this paper we presented experimental evidence that it is possible to dodge a Hopf supercritical bifurcation, provided that the system has two linearly stable regions on the two sides of the unstable one and that it is possible to directly control the bifurcation parameter and ramp it fast enough. The required ramp rate is a function of the system's parameters, and in particular the ramp time $T_{\mathrm{R}}$ needs to be shorter than the characteristic growth time $T_{v} \propto 1 / v_{\max }$, where $v_{\max }$ is the system's maximum linear growth rate in the bifurcation. We introduced an oscillator model which is able to reproduce the system dynamics in the transitory operation. This low-order model allowed us to develop a procedure to assess the risk of incurring in dangerous high-amplitude outputs of the system during the ramp, as a function of the system's parameters and of the ramp rate. These results can be applied to 
any system exhibiting a non-monotonic bifurcation, and provide a tool to estimate its useful life.

\section{ACKNOWLEDGMENTS}

This research is supported by the Swiss National Science Foundation under Grant 160579.

\section{REFERENCES}

\section{REFERENCES}

[1] Kuznetsov, Y. A., 2013. Elements of applied bifurcation theory, Vol. 112. Springer Science \& Business Media.

[2] Hoegh-Guldberg, O., Mumby, P. J., Hooten, A. J., Steneck, R. S., Greenfield, P., Gomez, E., Harvell, C. D., Sale, P. F., Edwards, A. J., Caldeira, K., et al., 2007. "Coral reefs under rapid climate change and ocean acidification". science, 318(5857), pp. 1737-1742.

[3] Bosi, S., and Desmarchelier, D., 2018. "Limit cycles under a negative effect of pollution on consumption demand: the role of an environmental kuznets curve". Environmental and Resource Economics, 69(2), pp. 343-363.

[4] Lenton, T. M., Held, H., Kriegler, E., Hall, J. W., Lucht, W., Rahmstorf, S., and Schellnhuber, H. J., 2008. "Tipping elements in the earth's climate system". Proceedings of the national Academy of Sciences, 105(6), pp. 1786-1793.

[5] KÃijhnen, J., Braunshier, P., Schwegel, M., Kuhlmann, H. C., and Hof, B., 2015. "Subcritical versus supercritical transition to turbulence in curved pipes". Journal of Fluid Mechanics, 770, May 10, p. 12.

[6] Scheffer, M., Bascompte, J., Brock, W. A., Brovkin, V., Carpenter, S. R., Dakos, V., Held, H., Van Nes, E. H., Rietkerk, M., and Sugihara, G., 2009. "Early-warning signals for critical transitions". Nature, 461(7260), p. 53.

[7] Scheffer, M., Carpenter, S. R., Lenton, T. M., Bascompte, J., Brock, W., Dakos, V., Van de Koppel, J., Van de Leemput, I. A., Levin, S. A., Van Nes, E. H., et al., 2012. "Anticipating critical transitions". science, 338(6105), pp. 344348.

[8] Kuehn, C., 2011. “A mathematical framework for critical transitions: Bifurcations, fast-slow systems and stochastic dynamics". Physica D: Nonlinear Phenomena, 240(12), pp. 1020-1035.

[9] Karnatak, R., Kantz, H., and Bialonski, S., 2017. "Early warning signal for interior crises in excitable systems". Physical Review E, 96(4), p. 042211.

[10] Dakos, V., Scheffer, M., van Nes, E. H., Brovkin, V., Petoukhov, V., and Held, H., 2008. "Slowing down as an early warning signal for abrupt climate change". Proceedings of the National Academy of Sciences, 105(38), pp. 14308-14312.
[11] Dakos, V., Carpenter, S. R., van Nes, E. H., and Scheffer, M., 2015. "Resilience indicators: prospects and limitations for early warnings of regime shifts". Philosophical Transactions of the Royal Society B: Biological Sciences, 370(1659), p. 20130263.

[12] Drake, J. M., and Griffen, B. D., 2010. "Early warning signals of extinction in deteriorating environments". Nature, 467(7314), p. 456.

[13] Ritchie, P., and Sieber, J., 2016. "Early-warning indicators for rate-induced tipping". Chaos: An Interdisciplinary Journal of Nonlinear Science, 26(9), p. 093116.

[14] Lenton, T., Livina, V., Dakos, V., Van Nes, E., and Scheffer, M., 2012. "Early warning of climate tipping points from critical slowing down: comparing methods to improve robustness". Phil. Trans. R. Soc. A, 370(1962), pp. 11851204.

[15] Nikolaou, A., Gutiérrez, P. A., Durán, A., Dicaire, I., Fernández-Navarro, F., and Hervás-Martínez, C., 2015. "Detection of early warning signals in paleoclimate data using a genetic time series segmentation algorithm". Climate Dynamics, 44(7-8), pp. 1919-1933.

[16] Bilinsky, L., and Baer, S., 2018. "Slow passage through a hopf bifurcation in excitable nerve cables: Spatial delays and spatial memory effects". Bulletin of mathematical biology, 80(1), pp. 130-150.

[17] Poinsot, T., 2017. "Prediction and control of combustion instabilities in real engines". Proceedings of the Combustion Institute, 36(1), pp. 1-28.

[18] Lieuwen, T. C., 2012. Unsteady combustor physics. Cambridge University Press.

[19] Ghirardo, G., Juniper, M. P., and Bothien, M. R., 2018. "The effect of the flame phase on thermoacoustic instabilities". Combustion and Flame, 187, pp. 165-184.

[20] Bonciolini, G., Ebi, D., Doll, U., Weilenmann, M., and Noiray, N., 2018. "Effect of wall thermal inertia upon transient thermoacoustic dynamics of a swirl-stabilized flame". Proceedings of the Combustion Institute.

[21] Li, L. K., and Juniper, M. P., 2013. "Lock-in and quasiperiodicity in hydrodynamically self-excited flames: Experiments and modelling". Proceedings of the Combustion Institute, 34(1), pp. 947-954.

[22] Terhaar, S., Ćosić, B., Paschereit, C., and Oberleithner, K., 2016. "Suppression and excitation of the precessing vortex core by acoustic velocity fluctuations: An experimental and analytical study". Combustion and Flame, 172, pp. 234251.

[23] Noiray, N., and Denisov, A., 2017. "A method to identify thermoacoustic growth rates in combustion chambers from dynamic pressure time series". Proceedings of the Combustion Institute, 36(3), pp. 3843-3850.

[24] Bonciolini, G., Boujo, E., and Noiray, N., 2017. “Outputonly parameter identification of a colored-noise-driven van- 
der-pol oscillator: Thermoacoustic instabilities as an example". Physical Review E, 95(6), p. 062217.

[25] Noiray, N., and Schuermans, B., 2013. "Deterministic quantities characterizing noise driven hopf bifurcations in gas turbine combustors". International Journal of NonLinear Mechanics, 50, pp. 152-163.

[26] Boujo, E., and Noiray, N., 2017. "Robust identification of harmonic oscillator parameters using the adjoint fokkerplanck equation". Proc. R. Soc. A, 473(2200), p. 20160894.

[27] Culler, W., Chen, X., Samarasinghe, J., Peluso, S., Santavicca, D., and OâĂŹConnor, J., 2018. "The effect of variable fuel staging transients on self-excited instabilities in a multiple-nozzle combustor". Combustion and Flame, 194, pp. 472-484.

[28] Bonciolini, G., Ebi, D., Boujo, E., and Noiray, N., 2018. "Experiments and modelling of rate-dependent transition delay in a stochastic subcritical bifurcation". Royal Society open science, 5(3), p. 172078.

[29] Miller, N. J., and Shaw, S. W., 2012. "Escape statistics for parameter sweeps through bifurcations". Physical Review E, 85(4), p. 046202.

[30] Hu, Z., Cheng, L., and Berne, B., 2010. "First passage time distribution in stochastic processes with moving and static absorbing boundaries with application to biological rupture experiments". The Journal of chemical physics, 133(3), p. 034105.

[31] Ritchie, P., and Sieber, J., 2017. "Probability of noiseand rate-induced tipping". Physical Review E, 95(5), p. 052209.

[32] Berglund, N., and Gentz, B., 2002. "Beyond the fokkerplanck equation: Pathwise control of noisy bistable systems". Journal of Physics A: Mathematical and General, 35(9), p. 2057. 